\section{BEWARE UNSCRUPULOUS WEBSITES}

Southern Dental Industries (SDI) are advising all customers to only buy SDI products through their usual dental dealer and not to be taken in by companies offering bargain basement prices. The following companies only are authorised by SDI: BDSI, Henry Schein Minerva, Budget, Kent Express, Claudius Ash, BF Mulholland, Click Dental, Oraldent, Dental Health Boutique, Precision Dental, Dental Sky, Skillbond, Dental Supply House, Survival, Dentaquip, The Dental Directory, Trycare Dental Linkline, Wright Cottrell.

There are unscrupulous websites out there who are allegedly selling products branded as Pola. SDI cannot guarantee the authenticity of products sold through unregulated dealers. They may have similar packaging, carry the

\section{NEW CONSUMER INSURANCE}

Bupa is introducing a consumer dental insurance product for the first time, in response to high demand.

Dental Cover 10 and 20 from Bupa allow customers to choose whichever dentist they want to visit. Dental Cover 10 offers customers a full refund for NHS treatment (bands 1-3) and market-leading oral cancer cover up to $£ 15,000$ per person per year (waiting periods apply).

Dental Cover 20 allows customers with a privately registered dentist to

\section{pola SDI

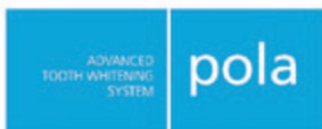 \\ Whiter. Brighter. You.}

SDI logo and look the same, but unless you buy your Pola products through your regular dental dealer you may be putting your patients at risk.

SDI's Pola Advanced Tooth Whitening System is only sold to registered dental professionals via authorised dental dealers.

Reader response number $\mathbf{6 1}$

\section{CLEAN, PROTECTED, WHITE}

Removing stains in just one minute, with over $90 \%$ of staining removed over a five minute period, the Beverly Hills Formula range of whitening toothpastes offer a solution for all common dental problems.

Patients affected by tooth sensitivity should use Sensitive Whitening Expert or Total Enamel Sensitive Expert which contains desensitising agents to effectively reduce the pain of sensitive teeth. To prevent the causes and effects of gum disease Dentist's Choice Gum \& Whitening Expert is recommended, claim up to $£ 150$ a year for routine treatment such as check-ups, X-rays and scale and polish. For more expensive, non-cosmetic treatment such as fillings, root canals, extractions, crowns, bridgework and dentures, customers can claim up 75\% of each bill, with up to $£ 700$ paid back per year. Cover also includes emergency dental treatment and treatment for dental injury (waiting periods apply).

With no underwriting or pre-enrolment checks required, set-up is quick and easy for the customer. Reader response number 62

\section{NOT JUST ANY CAMERA}

For practices seeking to offer their patients something a little bit different, the ANY intraoral camera from The Dental Directory is ideal.

The ANY intraoral camera is a multi-purpose device that can be plugged directly into a television set to allow patients to check their oral health from home. While ANY is certainly not suitable for patient selfdiagnosis, it can be a powerful tool for increasing oral health awareness.

From the patient's perspective the ANY intraoral camera is incredibly simple and easy to use. Similar to a toothbrush in design ANY is both comfortable and familiar to hold, and can be used with disposable covers to prevent cross infection.

ANY intraoral cameras are available exclusively from The Dental Directory in display boxes of five.

Reader response number 63

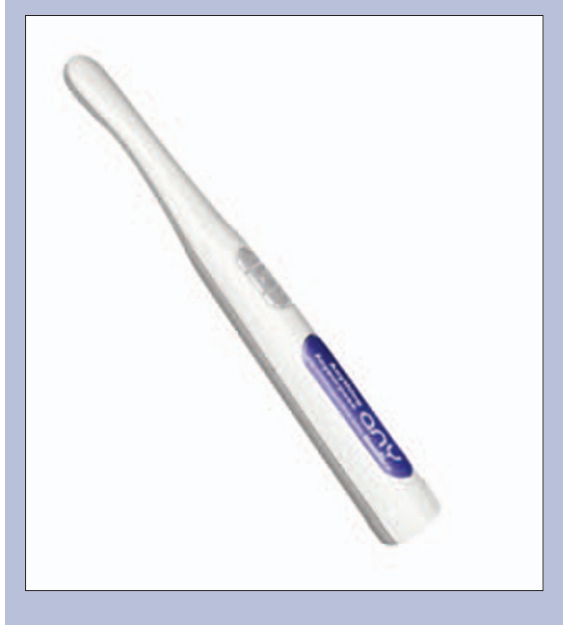

boasting a unique gum protection system to invigorate and strengthen the gums and stop bleeding. Those

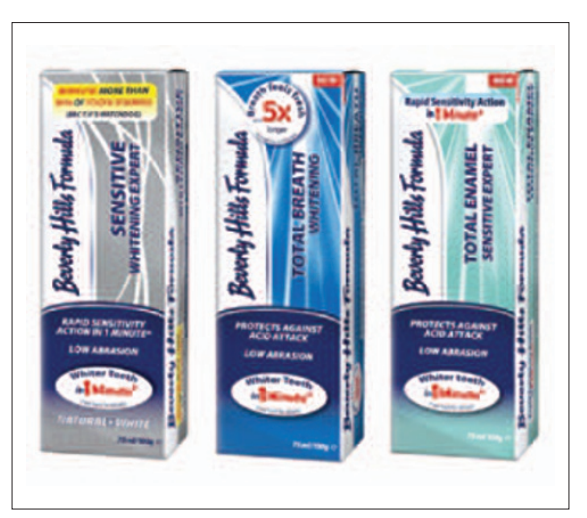

suffering from bad breath would benefit from Total Breath Whitening, providing a great minty flavour for long-lasting fresh breath up to five times longer.

The preventive qualities found in Total Protection Whitening are synonymous throughout the range, plus a recent study confirms that Beverly Hills Formula whitening toothpaste is less abrasive than other leading brands of both whitening and regular toothpastes, helping restore your patients' teeth to a natural white colour, safely, quickly and effectively. Reader response number 64 